\title{
Attentional spreading in object-based attention: The roles of target-object integration and target presentation time
}

\author{
Jingjing Zhao • Feng Kong • Yonghui Wang
}

Published online: 5 March 2013

(C) Psychonomic Society, Inc. 2013

\begin{abstract}
What is the best account to explain the objectbased attentional benefit - that is, the spread of attention within an attended object or prioritization of search across possible target locations within an attended object? Using a task in which the location of the target was known with certainty, in the present study we systematically manipulated the type (letters or bites) and the presentation time (long or short) of the target and flankers in order to test the effects of target-object integration and target presentation time on object-based attention. The results showed that an objectbased effect could appear when the target was a bite, no matter whether the target presentation time was long or short; but when the target was a letter, an object-based effect was only observed when the target presentation time was short enough. These findings provide additional evidence supporting the argument of attentional spreading in object-based attention. However, this spreading is moderated jointly by targetobject integration and the target presentation time.
\end{abstract}

Keywords Object-based attention · Attentional spreading · Search prioritization · Target-object integration $\cdot$ Target presentation time

It has been well supported that the visual system is unable to perceive an entire scene with equal degrees of detailed description in all parts. In order to perceive the complex visual world effectively, humans can select a portion in a

J. Zhao $(\bowtie) \cdot$ F. Kong $\cdot$ Y. Wang $(\bowtie)$

School of Psychology, Shaanxi Provincial Key

Laboratory of Behavior \& Cognitive Neuroscience,

Shaanxi Normal University, Xi' an, China

e-mail: zhaojingjing_31@126.com

e-mail: wyonghui@snnu.edu.cn

F. Kong

State Key Laboratory of Cognitive Neuroscience and Learning,

Beijing Normal University, Beijing, China scene for detailed processing with selective attention. A large body of literature on visual attention has emphasized the spatial nature of the attentional selection (e.g., C. W. Eriksen \& Hoffman, 1972; Posner, Snyder, \& Davidson, 1980). However, considerable evidence exists that attentional selection can also be based on objects that are present in the environment. The latter perspective has gained ground after several studies demonstrated that two or more features belonging to a single object are identified more quickly and more accurately than features belonging to different objects, even when the different objects are superimposed spatially (Behrmann, Zemel, \& Mozer, 1998; Duncan, 1984; Egly, Driver, \& Rafal, 1994; Goldsmith \& Yeari, 2003; Kliegl, Wei, Dambacher, Yan, \& Zhou, 2010); this is called an object-based effect or benefit. Two possible accounts have been proposed to interpret this object-based effect (Cepeda \& Kramer, 1999; Chen \& Cave, 2006, 2008; Ho, 2011; Hollingworth, Maxcey-Richard, \& Vecera, 2012; Müller \& Kleinschmidt, 2003; Richard, Lee, \& Vecera, 2008; Shomstein \& Behrmann, 2008; Shomstein \& Yantis, 2002, 2004).

The first of these accounts is the attentional-spreading account (Chen \& Cave, 2006, 2008; Desimone \& Duncan, 1995; Ho, 2011; Richard et al., 2008), which suggests that attending to an object guided by a spatial cue or task instructions involves spreading attention across the entire object; this spread of attention is limited by the contours and boundaries of the attended object (Desimone \& Duncan, 1995; Moore \& Fulton, 2005). Higher-level object structure feeds back into earlier visual areas, enhancing perceptual representation of the locations, which comprise the attended object (see also Mozer, 2002). Consequently, targets appearing on an attended object are processed more rapidly and accurately than are targets appearing elsewhere. Recently, Hollingworth et al. (2012) suggested that attention could form a gradient within the attended object when spreading, with the highest sensitivity at the cued location, 
and gradually decreasing sensitivity with increasing distance from the cue.

The second is the search prioritization account (e.g., Shomstein \& Behrmann, 2008; Shomstein \& Yantis, 2002, 2004), which proposes that the attended object is processed ahead of unattended objects and, more specifically, that currently unattended locations or features of an attended object will be searched ahead of currently unattended locations or features of an unattended object. One consequence of prioritization is that if a target location is certain, search is not necessary; thus, object-based attentional effects will be eliminated. To verify this assumption, Shomstein and Yantis (2002, Exps. 1-4) adopted a modified flanker compatibility paradigm (B. A. Eriksen \& Eriksen, 1974), in which the target letter was always presented in the center of the screen, with two flanker letters being aligned either vertically or horizontally. Specifically, the flanker letters could appear either in the same object as the target or in different objects. Their results indicated that whether the target and the flankers were in the same or different objects had no influence on the flanker compatibility effects, supporting the search prioritization account.

However, in some studies (Chen \& Cave, 2006, 2008; Ho, 2011; Richard et al., 2008), when the target location was known in advance with $100 \%$ positional certainty (e.g., Shomstein \& Yantis, 2002), attention could spread within the object that the target always occupied, thus incurring object-based effects. The difference in results between these studies and Shomstein and Yantis (2002) may have been due to two reasons. First, the highly familiar letter stimuli may have allowed for parsing of the targets and flankers away from the background objects, such that the objects were irrelevant to the task. This would explain why objectbased effects were not found in this paradigm. Second, the exposure duration of the stimuli may have been long enough to allow for this parsing away of letters from objects, again rendering objects irrelevant.

Richard et al. (2008) showed that the simple letters used in Shomstein and Yantis (2002), which could not integrate with the background objects, might not have needed perceptual-level attention to spread to these stimuli so that they could be distinguished from one another; however, for the stimuli that could integrate easily with the background objects (e.g., bites), attention might more likely be forced to select the object via attentional spreading, since the stimuli could not be selected independently of the object.

Another factor that likely influences object-based effects is target presentation time. Using simple letters but a very short target presentation time, other researchers have observed an object-based effect (e.g., $120 \mathrm{~ms}$ in Chen \& Cave, 2006, 2008; $51 \mathrm{~ms}$ in Ho, 2011). However, in Shomstein and Yantis (2002), an object-based effect was not found with the long target presentation time. The reason might be that the letters could not group perceptually with the objects; shortening the target and flanker duration might prevent the initiation of automated letter processing, so that the letters have no time to be parsed away from the objects, thus allowing attentional spreading to occur. Conversely, a long presentation time for the target and flankers might make the participants concentrate their attention on the task-related letters only, after an initial spread of attention across the entire object.

The goal of the present study was to demonstrate the significance of the target presentation time in object-based attention and the effect of target presentation time on the relationship between target-object integration and the object-based effect. To this end, we used two different types of stimuli (bites vs. letters) and two different target presentation times (long vs. short). In Experiment 1, we employed a paradigm similar to the one used by Shomstein and Yantis (2002), except that the presentation time of the target and the flankers was very long in Experiment $1 \mathrm{~A}$ and very short in Experiment 1B. The aim was to verify whether the presentation time of the target and the flankers could influence the object-based effect when the target and the flankers were letters. On the basis of the previous studies, it was predicted that object-based modulation of flanker interference would not be found when the target presentation time was long, but that it could be found when the presentation time was very short. In Experiment 2, we presented the rectangles for 1,000 ms in Experiment 2A and 1,040 ms in Experiment 2B (i.e., the rectangles' preview time), followed by the target and the flankers for $160 \mathrm{~ms}$ in Experiment $2 \mathrm{~A}$ and $120 \mathrm{~ms}$ in Experiment 2B, to make the exposure durations of the rectangles (i.e., the rectangles' preview time plus the presentation time of the target and flankers) equal. The aim was to rule out an alternative explanation, that the crucial factor that led to the emergence of the object-based effect in Experiment 1B was the exposure duration of the rectangles, which also decreased when the presentation time of the target and flankers was shortened. If the exposure duration of the rectangles contributed to the emergence of the object-based effect, then the results of Experiments $2 \mathrm{~A}$ and $2 \mathrm{~B}$ should be the same. If, on the other hand, the target presentation time predicted the emergence of the object-based effect, the results of Experiments $2 \mathrm{~A}$ and $2 \mathrm{~B}$ should be different. In Experiment 3, we employed a paradigm similar to the one used by Richard et al. (2008), except that the target presentation time was very long in Experiment $3 \mathrm{~A}$ and very short in Experiment 3B. The aim was to explore the role of target presentation time in objectbased attention when the target and flankers could integrate easily with the background rectangles (i.e., "bites" in Richard et al., 2008) and whether the target presentation time plays a different role in the object-based effect when the target and flankers could and could not integrate with the 
background objects. On the basis of previous studies, we predicted that when the targets and flankers could integrate easily with the background objects, an object-based effect would also be found at the short target presentation time, as in Experiment 1B. While, under this circumstance, there is no interference from other processes, attentional spreading could continue to play its role until the participants' response. That is, the results of Experiment 1 and 3 jointly showed that target presentation time moderated the relationship between targetobject integration and the object-based effect.

\section{Experiment 1}

\section{Method}

Participants A group of 34 Shaanxi Normal University students participated in Experiment 1 for extra credit in a psychology course-17 in Experiment $1 \mathrm{~A}$ and 17 in Experiment 1B. All of the participants reported normal or corrected-to-normal visual acuity and were naive as to the purpose of the experiment.

Apparatus and stimuli All stimuli were displayed on a 19in. color monitor. A chinrest maintained a fixed viewing distance of $62 \mathrm{~cm}$. A custom keyboard was used to collect buttonpress responses. Figure 1 shows the layout of some typical displays for the present experiment. The display
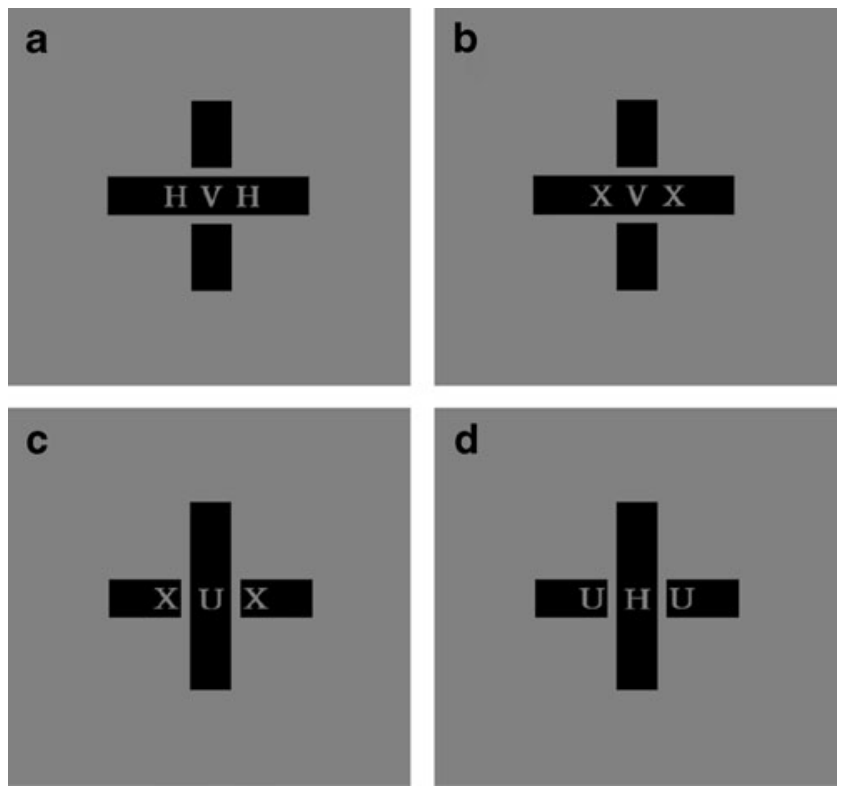

Fig. 1 Example display for each of the four conditions in Experiments 1 and 2: $\mathrm{H}$ and $\mathrm{V}$ required a left response; $\mathrm{V}$ and $\mathrm{X}$ required a right response. a Same object, compatible response. b Same object, incompatible response. c Different object, compatible response. d Different object, incompatible response. Flanker distance remained constant across the conditions consisted of three solid black rectangles that were presented on a square gray background region. The gray background square subtended $8^{\circ} \times 8^{\circ}$ of visual angle. The large central rectangle subtended visual angles of $2.8^{\circ} \times 0.5^{\circ}$, and the smaller flanking rectangles each subtended visual angles of $1^{\circ} \times 0.5^{\circ}$. The two smaller rectangles were located adjacent to the central rectangle with a gap of $0.1^{\circ}$. The long rectangle was oriented horizontally on a randomly selected half of the trials, and vertically on the remaining half.

A target letter always appeared in the center of the display, whereas flanking letters appeared adjacent to the target with a center-to-center distance of $0.18^{\circ}$, a distance that has been shown to yield robust flanker compatibility effects (e.g., B. A. Eriksen \& Eriksen, 1974). Each letter subtended $0.37^{\circ} \times 0.37^{\circ}$ of visual angle with the color of the gray background square. The flanking letters and the target letters were taken from the same set $(\mathrm{H}, \mathrm{V}, \mathrm{U}$, and $\mathrm{X})$.

Design and procedure A $2 \times 2 \times 2$ factorial design was used, with Target-Flanker Compatibility (compatible or incompatible) and Target-Flanker Relation (same or different objects) as within-subjects factors and Target Presentation Time (long or short) as a between-subjects factor. The target and flankers were considered compatible if they all mapped onto the same response key, and incompatible if they mapped onto different response keys (in the compatible condition, the target and flankers were always different letters). Each of the four combinations of conditions occurred equally often. Rectangle orientation and target and distractor identity were selected randomly and equally often within blocks, within the constraints of the experimental design. The participants were instructed to respond to the letters by using their index or middle finger to press one of the two designated keys on the keyboard (the "Z" key if the target was $\mathrm{H}$ or $\mathrm{V}$, and the " $\mathrm{M}$ " key if the target was $\mathrm{U}$ or X).

Each trial began with the gray background presented for a 1,000-ms intertrial interval (ITI). The three rectangles were then presented on the screen for $1,000 \mathrm{~ms}$, and the target and the flankers appeared. In Experiment 1A, the stimuli remained on the screen until the participant responded. In Experiment 1B, the target and the flankers were presented on the screen for $120 \mathrm{~ms}$, and then followed by a blank screen for the participants to respond (see Fig. 2). The participants were instructed to respond as quickly as possible while making no more than about $5 \%$ errors. The error feedback consisted of a beep and an increase in the ITI of $1,000 \mathrm{~ms}$ following that trial. Each participant completed one 48-trial practice block and eight blocks of 48 experimental trials, with short breaks in between. The entire experiment took approximately $20 \mathrm{~min}$ to complete. 
Fig. 2 Sequences of events in Experiments 1 and 2

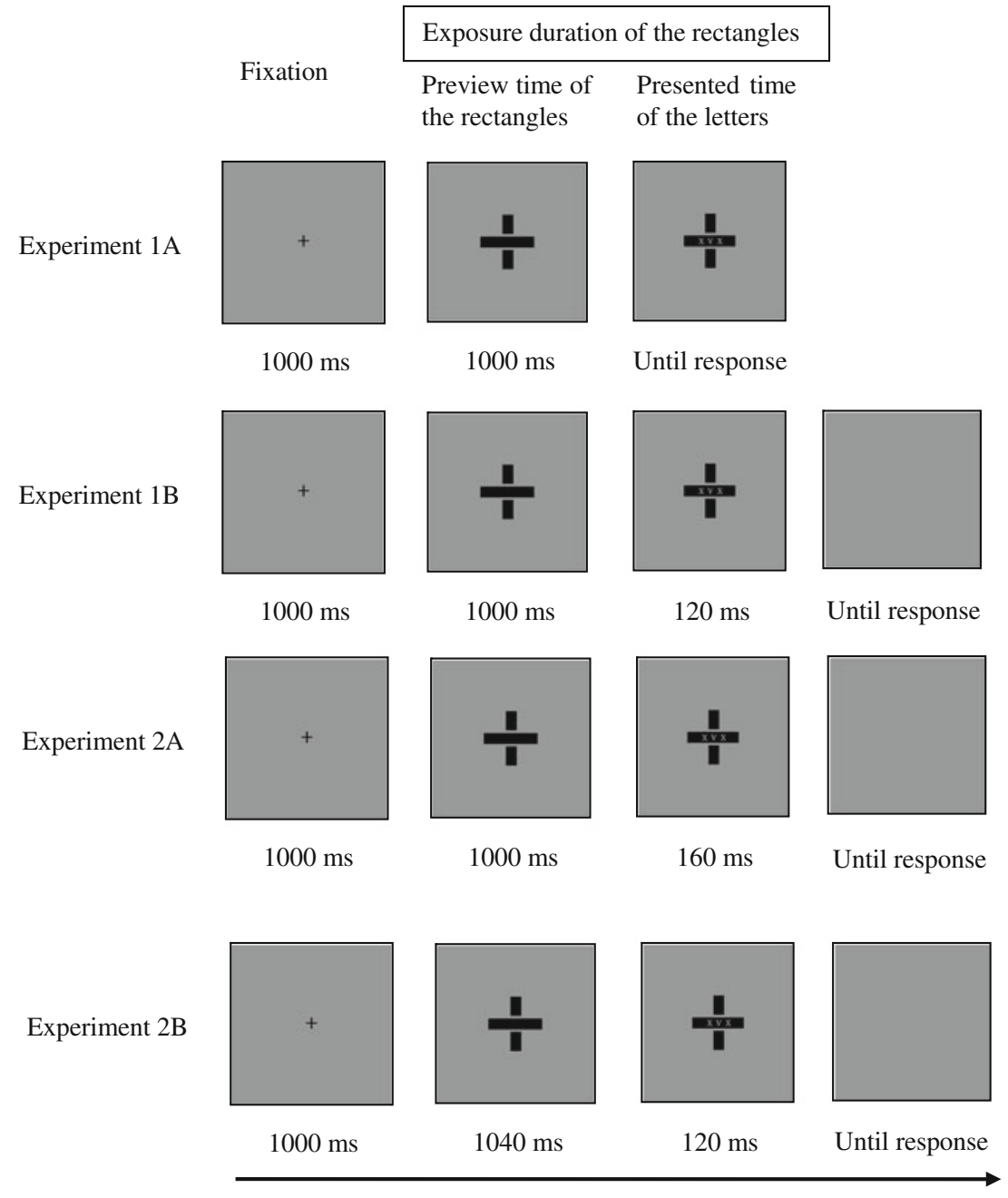

Experiment $1 A$ Figure 3 shows the mean correct RTs and the mean error rates for each condition of Experiment $1 \mathrm{~A}$. A repeated measures ANOVA was conducted, with Compatibility and Relation as within-subjects factors and RT and error rate as the dependent measures. The ANOVA revealed a significant main effect of compatibility $[F(1,16)=$ 33.61, $p<.001]$ : RTs for compatible flankers $(M=609 \mathrm{~ms})$ were faster than those for incompatible ones $(M=632 \mathrm{~ms})$. The main effect of relation and the interaction between compatibility and target-flanker relation were not significant $\left[F_{\mathrm{s}}(1,16)<1\right]$. The RTs were similar between same-object $(624 \mathrm{~ms})$ and different-object $(619 \mathrm{~ms})$ trials. The flanker interference effect of $23 \mathrm{~ms}$ did not depend on whether or not the target and flankers were within the same object.

An analysis of the error rates revealed a similar pattern. The main effect of compatibility was significant $[F(1,16)=$ $7.65, p<.05]$, and the main effect of relation was not significant $[F(1,16)<1]$. In addition, we found no significant interaction between the factors. All of the participants performed with at least $90 \%$ accuracy in each condition in all of the experiments, and there was no indication of a speed-accuracy trade-off. 
a

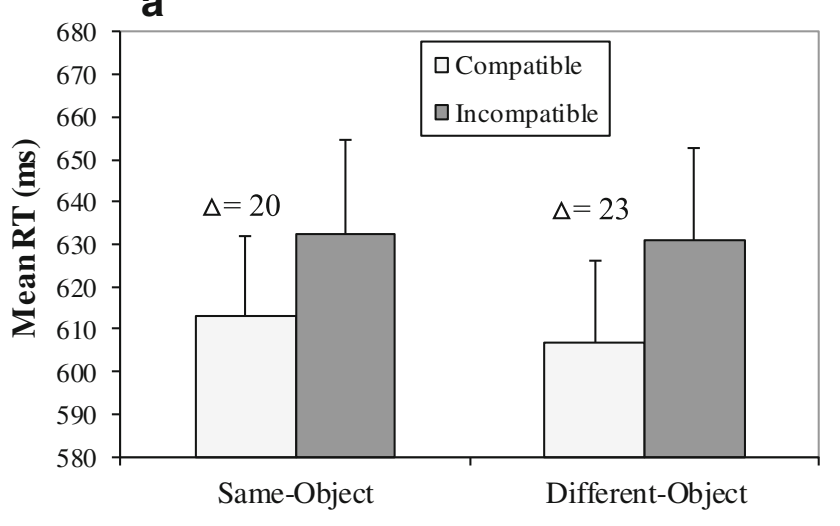

Relation

b

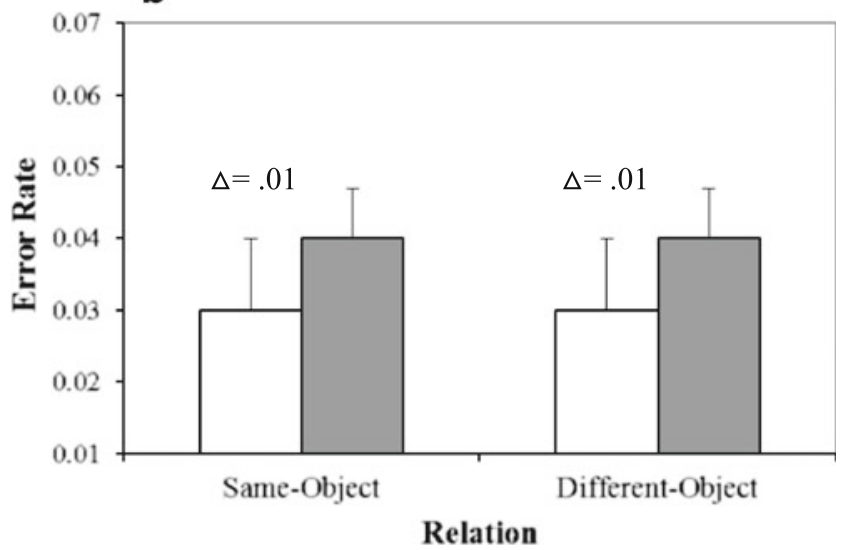

Fig. 3 Mean correct reaction times (a) and error rates (b) in Experiment 1A. The data are collapsed across vertical and horizontal orientations of the display. Error bars indicate the standard errors of the means

Experiment $1 B$ Figure 4 shows the mean correct RTs and the mean error rates for Experiment 1B. The data were subjected to a two-way ANOVA, with Relation and Compatibility as within-subjects factors and RT and error rate as dependent measures. The ANOVA revealed a significant main effect of compatibility $[F(1,16)=34.23$, $p<.001]$ : RTs were faster for compatible $(M=469 \mathrm{~ms})$ than for incompatible $(M=490 \mathrm{~ms})$ flankers. More importantly, the interaction between compatibility and relation was also significant $[F(1,16)=25.82, p<.001]$. Further analysis of this interaction effect indicated a larger compatibility effect when the target and flankers were in the same object than when they were in different objects $[t(16)=5.08, p<.001]$.

The analysis of error rates (see Fig. 4B) also revealed a significant main effect of compatibility $[F(1,16)=6.6$, $p<.05$ ], suggesting higher accuracy when the flankers were compatible than when they were incompatible. No other effects approached significance, and there was no indication of a speed-accuracy trade-off.

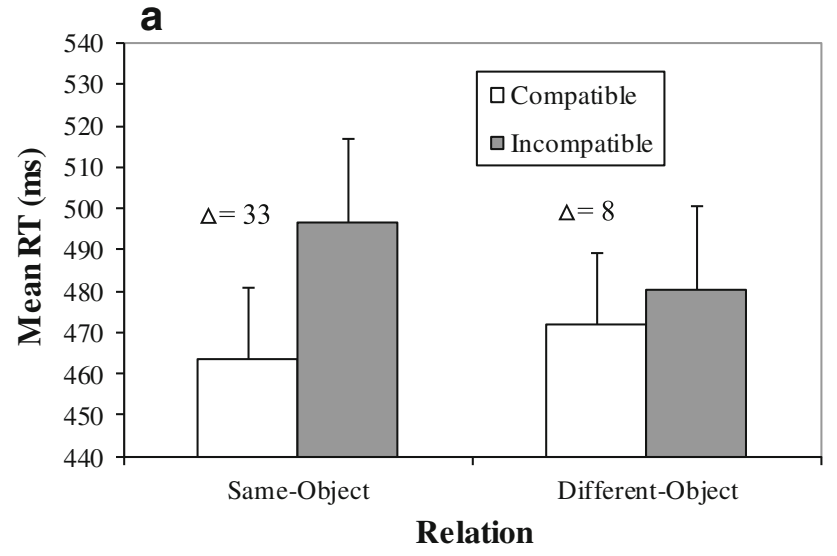

b

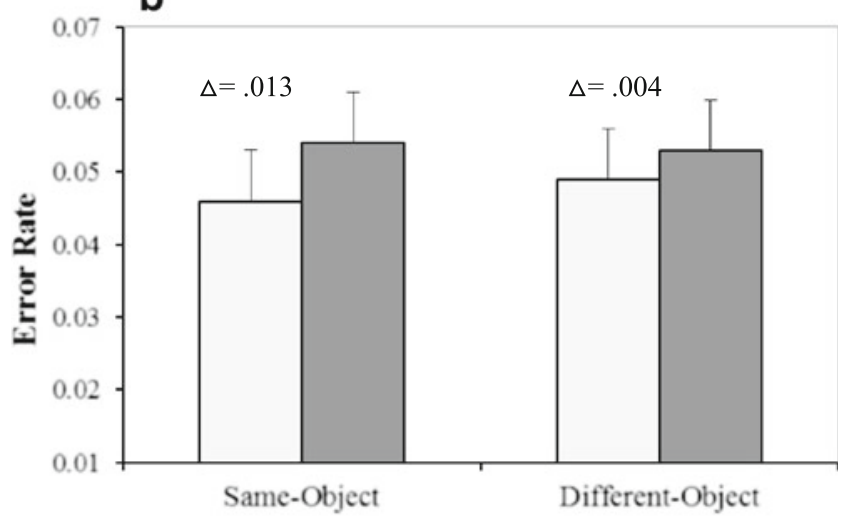

Relation

Fig. 4 Mean correct reaction times (a) and error rates (b) in Experiment 1B. The data are collapsed across vertical and horizontal orientations of the display. Error bars indicate the standard errors of the means

\section{Discussion}

The results of Experiment 1A replicated the findings of Shomstein and Yantis (2002, Exps. 1-4). Just as in their experiments, our study showed a response compatibility effect, suggesting that the irrelevant flankers impaired the ability to process the target efficiently. The most important result of Experiment $1 \mathrm{~A}$ was the absence of a Compatibility $\times$ Target-Flanker Relation interaction, indicating that the amounts of flanker interference were similar, regardless of whether the target and flankers were within the same object or in different objects. This result is consistent with the findings of Shomstein and Yantis (2002) and their statement of a search prioritization account of object effects. If object effects are the result of an object-based search prioritization strategy, as Shomstein and Yantis (2002) proposed, it is not surprising that no object-based effects were observed in our experiment, since such a strategy was not necessary.

However, in Experiment 1B, when the presentation time of the target and the flankers was very short, object-based 
modulation of flanker interference was observed. The participants showed greater interference from incompatible flankers when the flankers were in the same object as the target than when they were in different objects than the target. Recall that in this experiment, as well as in those of Shomstein and Yantis (2002), the target and the flankers were all highly familiar letters and were task-related as compared to the objects; according to Richard et al. (2008), processing of these letters was more automated than processing of the objects. Because the letters did not presumably group perceptually with the objects, shortening the target and flanker duration prevented the initiation of automatic letter processing, thus allowing attentional spreading to occur. Because the major difference between Experiments $1 \mathrm{~A}$ and $1 \mathrm{~B}$ was the presentation time of the target and the flankers, it seems reasonable to attribute the different results of Experiment $1 \mathrm{~B}$ to the reduction of the presentation time of the letters.

Although our data provide clear evidence in support of the attentional-spreading account, it would be beneficial to examine one other alternative interpretation: the possibility that the crucial factor that predicted the emergence of the object-based effect was the exposure duration of the rectangles, which also decreased when the presentation time of the target and the flankers was shortened. Experiments $2 \mathrm{~A}$ and $2 \mathrm{~B}$ tested this alternative hypothesis by varying the presentation time of the target and the flankers and the preview time of the rectangles.

\section{Experiment 2}

\section{Method}

Participants A group of 35 students from the Shaanxi Normal University participated in Experiment 2 in order to fulfill a requirement in a psychology course; 17 participated in Experiment 2A and 18 in Experiment 2B. None of the participants participated in other experiments in this study. All of them reported normal or corrected-to-normal visual acuity and were naive as to the purpose of the experiment.

Apparatus and stimuli The stimuli were the same as those in Experiment 1.

Design and procedure The design and procedure were the same as in Experiment 1B, with the only difference being that, in Experiment 2A, the three rectangles were presented on the screen for $1,000 \mathrm{~ms}$ and then followed by the target and flankers for $160 \mathrm{~ms}$, and in Experiment 2B, the three rectangles were presented on the screen for 1,040 ms before the letters were presented for $120 \mathrm{~ms}$, thereby ensuring that in both experiments the rectangles were presented for equal amounts of time (see Fig. 2). All other elements of the design were kept the same as in Experiment 1B.

\section{Results}

Only the RTs for correct responses were analyzed. RTs less than $200 \mathrm{~ms}$ or greater than $2,000 \mathrm{~ms}$ were excluded from the analyses, resulting in the discarding of fewer than $1 \%$ of trials. A preliminary analysis indicated no main effect of the orientation of the rectangles and no interaction of orientation with any of the other factors (all $F_{\mathbf{S}}<1$ ), so the data were collapsed across the vertical and horizontal orientations.

A repeated measures ANOVA was conducted, with Compatibility and Relation as within-subjects factors, Experiment (2A or $2 \mathrm{~B})$ as a between-subjects factor, and RT and error rate as the dependent measures. The ANOVA revealed a significant main effect of compatibility $[F(1,33)=38.38, p<.001]$ : RTs for compatible flankers ( $M=455 \mathrm{~ms})$ were faster than those for incompatible ones $(M=473 \mathrm{~ms})$. More importantly, the three-way interaction of compatibility, relation, and experiment was also significant $[F(1,33)=9.78, p<.01]$. An analysis of the error rates revealed a similar pattern.

Experiment $2 A$ Figure 5 shows the mean correct RTs and the mean error rates for each condition of Experiment 2A. The data were subjected to a two-way ANOVA, with Relation and Compatibility as within-subjects factors and $\mathrm{RT}$ and error rate as the dependent measures. The ANOVA revealed a significant main effect of compatibility $[F(1,16)=19.32, p<.001]$ : RTs were faster for compatible ( $M=427 \mathrm{~ms})$ than for incompatible $(M=442 \mathrm{~ms})$ flankers. In addition, we found a significant main effect of relation $[F(1,16)=4.92, p=.04]$, so that RTs were slightly slower when the target and flankers appeared in the same object $(M=439 \mathrm{~ms})$ than when they appeared in different objects $(M=429 \mathrm{~ms})$. However, the interaction between compatibility and relation was not significant $[F(1,16)<1]$. The analysis of the error rates revealed the same pattern.

Experiment $2 B$ Figure 6 shows the mean correct RTs and the mean error rates for each condition of Experiment 2B. An ANOVA was conducted, with Relation and Compatibility as within-subjects factors and RT and error rate as the dependent measures. The ANOVA revealed a significant main effect of compatibility $[F(1,17)=19.81$, $p<.001]$ : RTs were faster for compatible $(M=484 \mathrm{~ms})$ than for incompatible $(M=502 \mathrm{~ms})$ flankers. More importantly, the interaction between compatibility and relation was significant $[F(1,17)=14.17, p=.002]$. Further analysis of this interaction effect indicated a larger compatibility effect when the target and flankers 
a

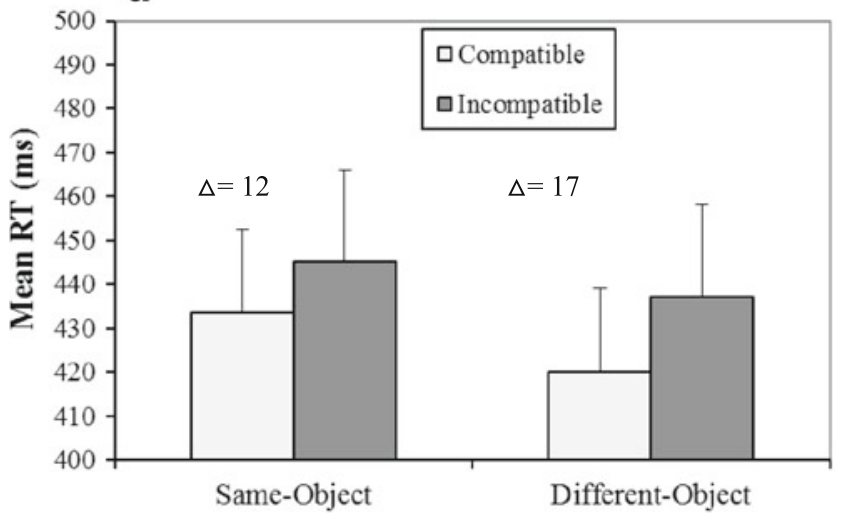

Relation

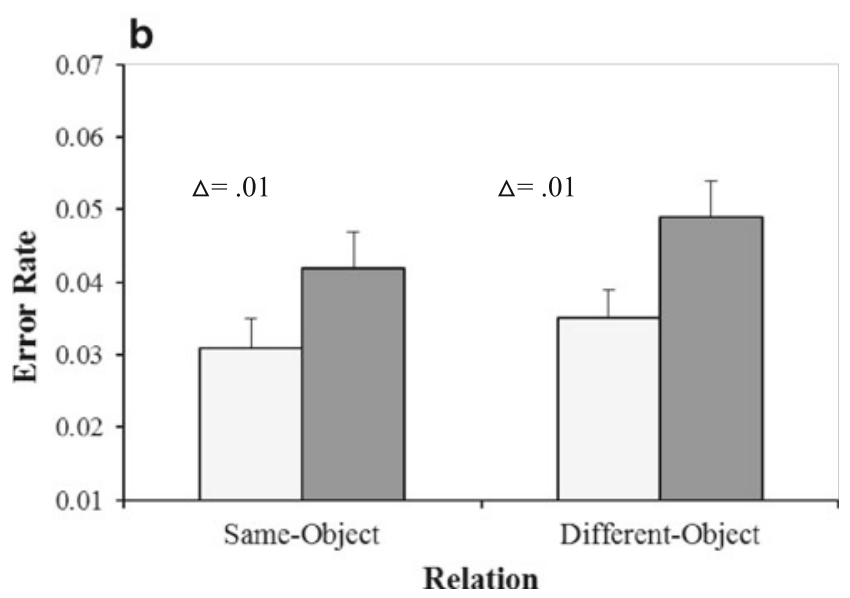

Fig. 5 Mean correct reaction times (a) and error rates (b) in Experiment 2A. The data are collapsed across vertical and horizontal orientations of the display. Error bars indicate the standard errors of the means

were in the same object than when they were in different objects $[t(17)=3.77, p=.002]$.

The analysis of error rates (see Fig. 6B) also revealed a significant main effect of compatibility $[F(1,17)=22.13$, $p<.001]$, suggesting higher accuracy when the flankers were compatible than when they were incompatible. The interaction between compatibility and relation was also significant $[F(1,17)=4.67, p<.05]$. Further analysis of this interaction effect indicated a larger compatibility effect when the target and flankers were in the same object than when they were in different objects $[t(17)=2.16, p<.05]$. No other effects approached significance, and there was no indication of a speed-accuracy trade-off.

\section{Discussion}

The results obtained in Experiments $2 \mathrm{~A}$ and $2 \mathrm{~B}$ revealed that it was the presentation time of the target and flankers, and not the exposure duration of the three rectangles, that modulated the object-based effect. The interaction between
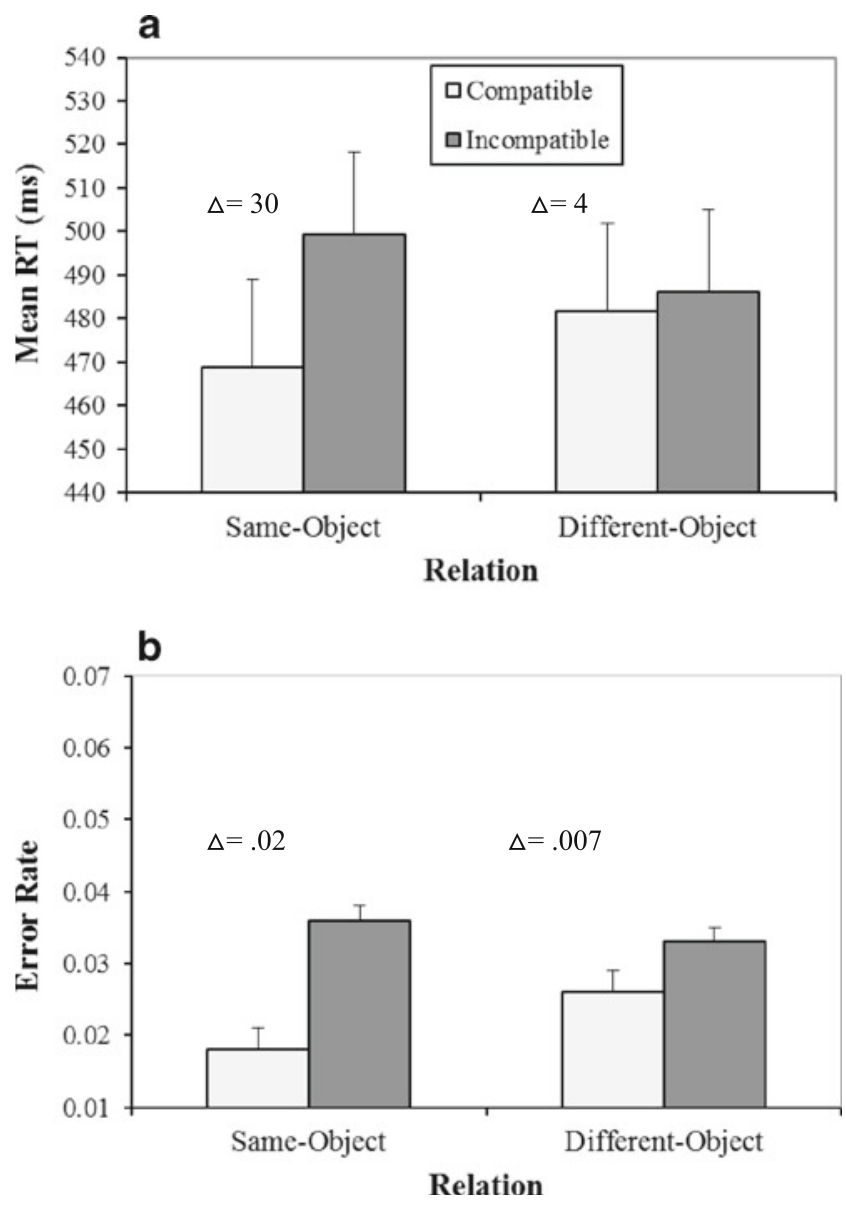

Fig. 6 Mean correct reaction times (a) and error rates (b) in Experiment 2B. The data are collapsed across vertical and horizontal orientations of the display. Error bars indicate the standard errors of the means

compatibility and relation was significant when the target presentation time was $120 \mathrm{~ms}$ (as it was in Exp. 1B) in Experiment 2B, supporting the attentional-spreading account, and the interaction was not significant when the presentation time was $160 \mathrm{~ms}$ in Experiment 2A, supporting the search prioritization account. These results were obtained even when the rectangles in Experiments 2A and 2B were always presented for a total of $1,160 \mathrm{~ms}$, indicating that the exposure duration of the rectangles did not impact on the data. The analysis of RT data in Experiments 1 and 2A showed that increasing the presentation time of the target and the flankers made the object-based effect disappear.

As we mentioned above, automatic processing of the letters prevented attentional spreading from continuing to play its role at a long target duration. Therefore, if there were no interference from this process (i.e., the targets and flankers could integrate easily with the objects), how would the target presentation time affect object-based attention? Would there be a moderating effect of target presentation time on the relationship between target-object integration 
and the object-based effect, just as we expected? Experiment 3 was designed to test this assumption.

\section{Experiment 3}

\section{Method}

Participants A group of 34 new students from the Shaanxi Normal University participated in Experiment 3 in order to fulfill a requirement in a psychology course; 17 participated in Experiment 3A and 17 in Experiment 3B. No participants had participated in other experiments in this study, and all of them reported normal or corrected-to-normal visual acuity and were naive as to the purpose of the experiment.

Apparatus and stimuli The apparatus was the same as in Experiments 1 and 2. Figure 7 shows the layout of some typical displays for the present experiment. The display consisted of one large or three small black rectangular objects, which were presented in the center of a square gray background region. The large rectangle subtended visual angles of $9.6^{\circ} \times 1.2^{\circ}$, and the smaller rectangles each subtended visual angles of $2.8^{\circ} \times 1.2^{\circ}$. The three smaller rectangles were located adjacent to each other, with a gap of $0.6^{\circ} \times 1.2^{\circ}$. The rectangles were oriented horizontally on a randomly selected half of the trials, and vertically on the remaining half.
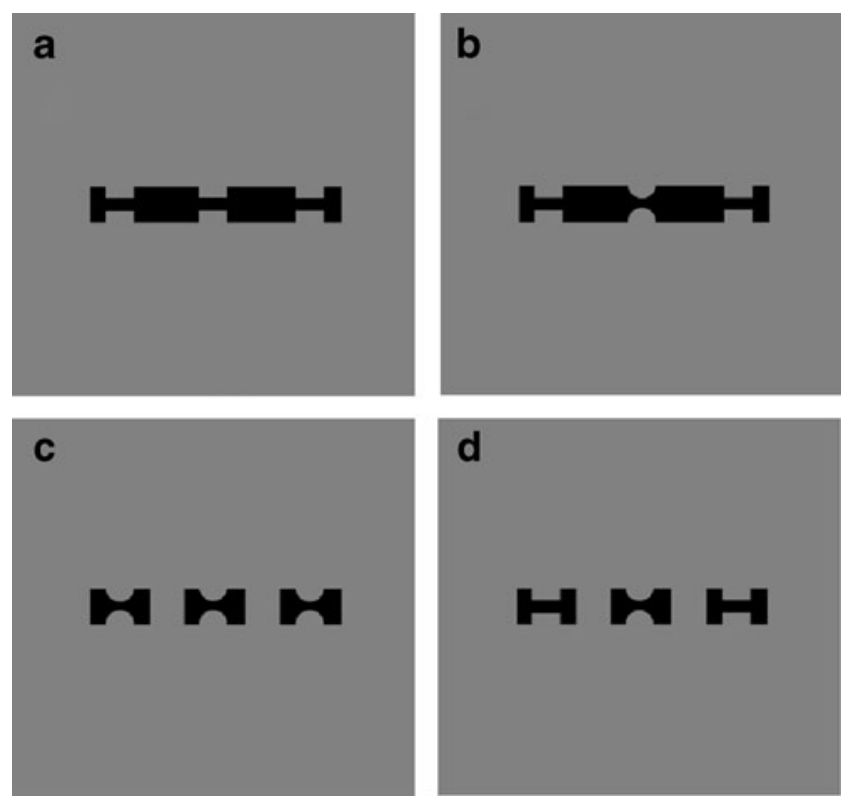

Fig. 7 Example displays for the four conditions in Experiment 3: A circular bite required a left response; a rectangular bite required a right response. a Same object, compatible response. b Same object, incompatible response. c Different object, compatible response. d Different object, incompatible response. The flanker distance remained constant across conditions
A target bite always appeared at the center of the display, whereas the far edges of the flanker bites were $3.9^{\circ}$ from the center of the screen. Each bite subtended $1^{\circ} \times 0.4^{\circ}$ of visual angle, with the color of the gray background square.

Design and procedure The design and procedure were same as in Experiment 1, with the only difference being that the target and the flankers were bites (see Fig. 8). The participants were instructed to respond to the bites on the "Z" key if the target was circular and the " $\mathrm{M}$ " key if the target was rectangular. All other elements of the design were kept the same as in Experiment 1.

\section{Results}

Only the RTs for correct responses were analyzed. RTs less than $150 \mathrm{~ms}$ or greater than $2,000 \mathrm{~ms}$ were excluded from the analyses, resulting in the discarding of fewer than $1 \%$ of the trials. A preliminary analysis indicated no main effect of the orientation of the rectangles and no interaction of orientation with any of the other factors (all $F_{\mathrm{S}}<1$ ), so the data were collapsed across the vertical and horizontal orientations.

A repeated measures ANOVA was conducted, with Compatibility and Relation as within-subjects factors, Target Presentation Time as a between-subjects factor, and RT and error rate as the dependent measures. The ANOVA revealed a significant main effect of compatibility $[F(1,32)=88.90, p<.001]$ : RTs for compatible flankers $(M=400 \mathrm{~ms})$ were faster than those for incompatible ones $(M=416 \mathrm{~ms})$. The main effect of relation $[F(1,32)=7.29, p<.05]$ and the Compatibility $\times$ Relation interaction $[F(1,32)=29.78, p<.001]$ were both significant. More importantly, the three-way interaction of compatibility, relation, and target presentation time was not significant $[F(1,32)<1, p>.05]$. These results showed that no matter whether the target presentation time was long or short, the interaction between compatibility and relation was significant, but the difference between the sizes of these interactions at the two target durations was not significant. An analysis of the error rates revealed a similar pattern. Figures 9 and 10 show the mean correct RTs and the mean error rates for each condition of Experiments $3 \mathrm{~A}$ and $3 \mathrm{~B}$, respectively.

\section{Discussion}

The results obtained in Experiments $3 \mathrm{~A}$ and $3 \mathrm{~B}$ revealed that the object-based effect was found when the target and flankers were "bites" for both long and short target presentation times. These results supported the attentionalspreading account and are consistent with those of preceding studies, which indicated that attentional spreading 
Fig. 8 Sequences of events in Experiment 3

occurred at an early stage (e.g., Kasai, 2010; Martinez, Ramanathan, Foxe, Javitt, \& Hillyard, 2007). Moreover, this effect could continue to play a role until participants'

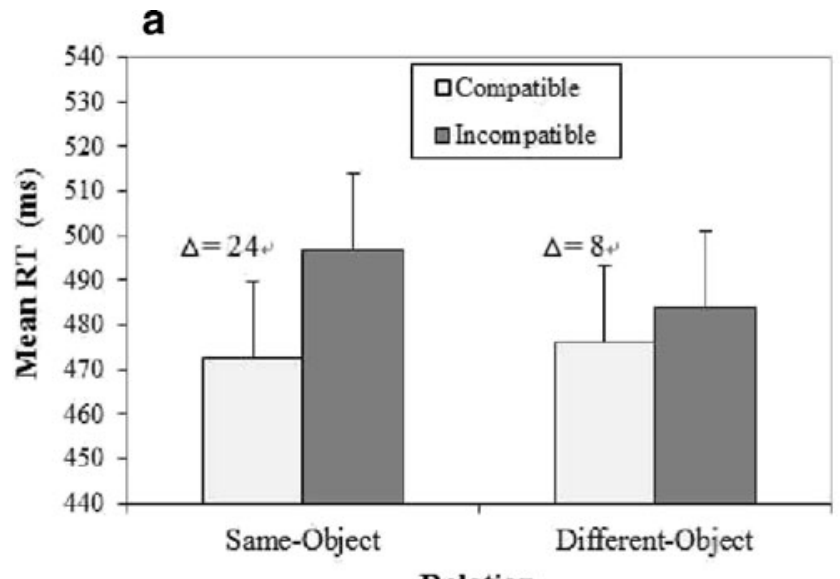

Relation

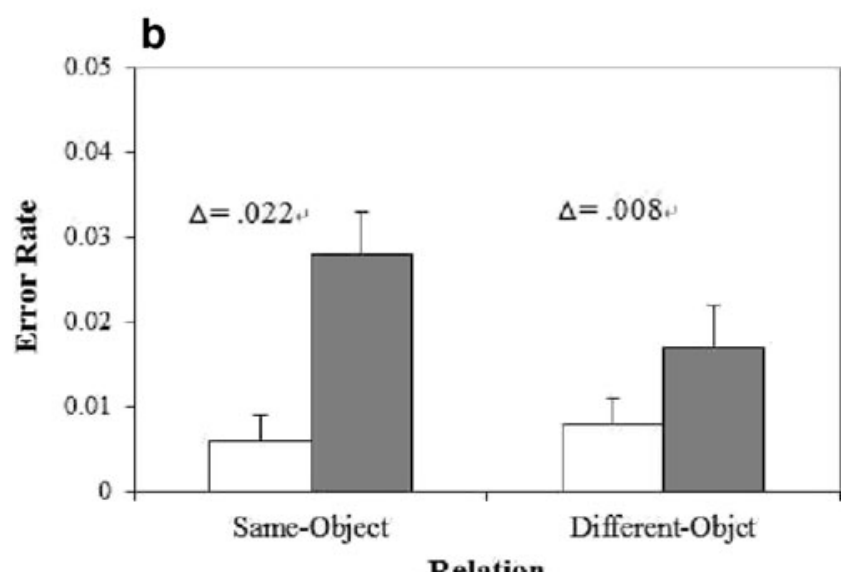

Fig. 9 Mean correct reaction times (a) and error rates (b) in Experiment 3A. The data are collapsed across vertical and horizontal orientations of the display. Error bars indicate the standard errors of the means
Exposure duration of the rectangles

Fixation $\begin{array}{ll}\begin{array}{l}\text { Preview time of } \\ \text { the rectangles }\end{array} & \begin{array}{l}\text { Presented time } \\ \text { of the letters }\end{array}\end{array}$
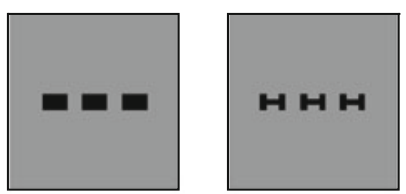

$1000 \mathrm{~ms}$

Until response

$1000 \mathrm{~ms}$
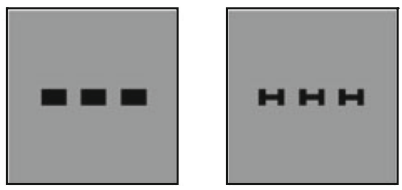

$1000 \mathrm{~ms}$

$1000 \mathrm{~ms}$

$120 \mathrm{~ms}$

Until response responses, since there was no interference from other processes when the target and the flankers integrated easily with the background objects. The results from

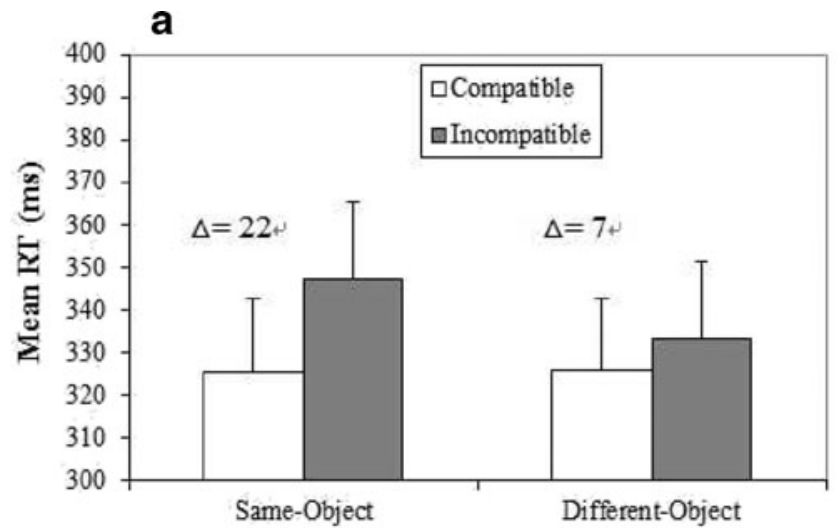

Relation

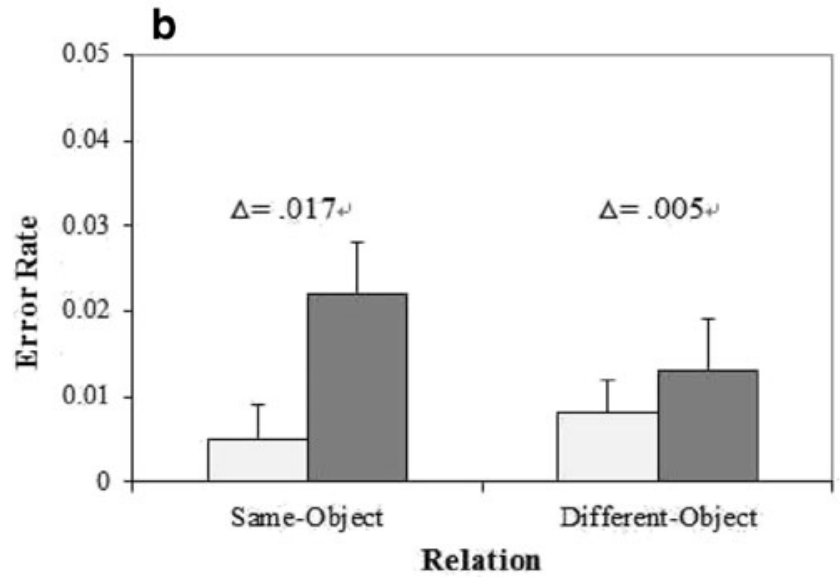

Fig. 10 Mean correct reaction times (a) and error rates (b) in Experiment 3B. The data are collapsed across vertical and horizontal orientations of the display. Error bars indicate the standard errors of the means 
Experiments 1 and 3 indicated that target presentation time moderated the relationship between target-object integration and the object-based effect: An object-based effect was found when the target was a bite, no matter whether the target presentation time was long or short; but when the target was a letter, the effect was only observed when the target presentation time was short enough.

\section{General discussion}

The experiments above add to the body of evidence demonstrating that an object-based effect can appear or disappear depending on specific factors, and further outline these factors.

\section{Attentional spreading versus search prioritization}

In all of the experiments reported here, the target locations were $100 \%$ certain before they appeared, and the participant was not required to search. The fact that an object-based effect arose in these conditions in Experiments 1B, 2B, and 3 demonstrated that it still existed, even when search was unnecessary. These results provide converging evidence that the object-based effect arises because of the spread of attention across the entire object (Chen \& Cave, 2006, 2008; Ho, 2011; Richard et al., 2008), as well as providing another piece of evidence against a search prioritization account of object-based attention in such a focusedattention task (Shomstein \& Yantis, 2002).

The role of target-object integration

In this study, when the targets could not integrate with the background objects and were simple letters in Experiments $1 \mathrm{~B}$ and $2 \mathrm{~B}$, an object-based effect was found. These findings extend the results from Richard et al. (2008), in which the object-based effect was only found when the targets were bites. Without any searching for the targets, an object-based attentional effect was found when the targets either could or could not integrate with the objects, which illustrated that to a certain extent, the attentional spreading was universal. However, this spreading also had its specificity between different types of stimuli, manifesting that letters were influenced by the target presentation time, but bites were not.

The role of target presentation time

Experiment 1A replicated the results of Shomstein and Yantis (2002). However, the results from Experiment 1B demonstrated that the object-based effect still appeared when the target location was $100 \%$ certainty. The only difference was that the target presentation time in Experiment $1 \mathrm{~B}$ was
$120 \mathrm{~ms}$, which was much shorter than that in Experiment 1A. The results of Experiments $1 \mathrm{~A}$ and $1 \mathrm{~B}$ suggested that target presentation time could affect the emergence of the object-based attentional effect when the targets were letters. By ruling out an alternative interpretation, Experiments $2 \mathrm{~A}$ and $2 \mathrm{~B}$ further supported this hypothesis. According to the results above, target presentation time does play a role in object-based attention.

Our design differed from that of Chen and Cave (2006): They focused more on the role of participants' perceiving the objects as separate objects than on the role of target presentation time, whereas, in the present study, we emphasized the target presentation time. The difference between our design and that of Chen and Cave (2008) was that we manipulated the presentation time of the target instead of the preview time of the rectangles. However, our argument does not contradict Chen and Cave (2006, 2008). That is, perceiving objects as separate objects (figure-ground segmentation in Chen, 1998; Moore, Yantis, \& Vaughan, 1998) and having sufficient time for developing perceptual object representations (Ho, 2011; Law \& Abrams, 2002; Richard et al., 2008; Shomstein \& Yantis, 2002) are prerequisites for generating an object-based effect. Contrary to what was found by Ho (2011), the present research demonstrated that object-based attentional effects are caused by the target presentation time rather than by the type of measurement. Ho found an object-based benefit only in a data-limited accuracy-based flanker measure, rather than an RT-based measure. In our study, the findings showed that by shortening the target presentation time, the object-based effect was also observed in an RT-based measurement.

The moderating effect of target presentation time on the relationship between target-object integration and object-based effect

Several studies have illustrated the importance of target-object integration on attention to objects (e.g., Richard et al., 2008). The present results extend this work by demonstrating that target presentation time moderated this relationship between target-object integration and the object-based effect. With a short target presentation time, the object-based effect appeared, no matter whether the targets were letters (Exps. 1B and 2B) or bites (Exp. 3B). This result suggested that this effect was caused by a mechanism that occurred in the early stage of visual processing, consistent with previous evidence based on early components as indicators for attentional spreading (e.g., Kasai, 2010; Martinez et al., 2007). However, with the long target presentation time, the object-based effect appeared only when the targets were bites (Exp. 3A).

In order to explain our results, we cited the "integrality hypothesis" suggested by Richard et al. (2008), and added the role of target presentation time. When task-relevant 
features are integral to the objects in a display (e.g., Baylis \& Driver, 1992; Egly et al., 1994; Richard et al., 2008), object-based attention might more likely be forced to select the object via attentional spreading, because the features could not be selected independently from the object. The effect could continue playing a role until the participant responded because of the absence of interference from other processes. When features are not integral to the objects-for example, simple letters (e.g., Chen \& Cave, 2006, 2008; Ho, 2011; Hollingworth et al., 2012; Shomstein \& Yantis, 2002), which can be automatically separated from each other-an objectbased effect due to attentional spreading might be interrupted by more efficient task-related letter processing. Therefore, shortening the target and flanker duration might prevent the initiation of automatic letter processing, thus allowing attentional spreading to occur. However, long presentation times of the target and flankers might make the participants concentrate their attention on the task-related letters only, after an initial spread of attention across the entire object.

Although our present findings support the attentionalspreading account of the object-based effect, we would not argue that such an account can explain all attentional effects. The paradigm that we used was to investigate the object-based attentional effect by ruling out search prioritization. Therefore, we cannot exclude the possibility that search prioritization could play a role in the object-based effect when target location is not certain. As such, future research should be dedicated to devising a new paradigm that could investigate attentional spreading and search prioritization simultaneously, to further refine the account for the object-based effect.

Author Note This research was supported by grants from the Innovation Funds of Graduate Programs (2012CXS025) of Shaanxi Normal University to Jingjing Zhao and the Key Program of the 11th Five-year Plan of National Education Science (DBA070070) to Yonghui Wang. We thank Xiaolin Zhou and the anonymous reviewers for helpful comments on earlier versions of this work, and Emlily Ullberg for polishing the language of the final revision. Correspondence should be addressed to Yonghui Wang, School of Psychology, Shaanxi Normal University and Shaanxi Provincial Key Laboratory of Behavior \& Cognitive Neuroscience, Xi'an, China (e-mail: wyonghui@snnu.edu.cn).

\section{References}

Baylis, G. C., \& Driver, J. (1992). Visual parsing and response competition: The effect of grouping factors. Perception \& Psychophysics, 51, 145-162. doi:10.3758/BF03212239

Behrmann, M., Zemel, R. S., \& Mozer, M. C. (1998). Object-based attention and occlusion: Evidence from normal participants and a computational model. Journal of Experimental Psychology. Human Perception and Performance, 24, 1011-1036. doi:10.1037/00961523.24.4.1011

Cepeda, N. J., \& Kramer, A. F. (1999). Strategic effects on objectbased attentional selection. Acta Psychologica, 103, 1-19.
Chen, Z. (1998). Switching attention within and between objects: The role of subjective organization. Canadian Journal of Experimental Psychology, 52, 7-16.

Chen, Z., \& Cave, K. R. (2006). Reinstating object-based attention under positional certainty: The importance of subjective parsing. Perception \& Psychophysics, 68, 992-1003. doi:10.3758/ BF03193360

Chen, Z., \& Cave, K. R. (2008). Object-based attention with endogenous cuing and positional certainty. Perception \& Psychophysics, 70, 1435-1443. doi:10.3758/PP.70.8.1435

Desimone, R., \& Duncan, J. (1995). Neural mechanisms of selective visual attention. Annual Review of Neuroscience, 18, 193-222. doi:10.1146/annurev.ne.18.030195.001205

Duncan, J. (1984). Selective attention and the organization of visual information. Journal of Experimental Psychology. General, 113, 501-517. doi:10.1037/0096-3445.113.4.501

Egly, R., Driver, J., \& Rafal, R. D. (1994). Shifting visual attention between objects and locations: Evidence from normal and parietal lesion subjects. Journal of Experimental Psychology. General, 123, 161-177. doi:10.1037/00963445.123.2.161

Eriksen, B. A., \& Eriksen, C. W. (1974). Effects of noise letters upon the identification of a target letter in a nonsearch task. Perception \& Psychophysics, 16, 143-149. doi:10.3758/ BF03203267

Eriksen, C. W., \& Hoffman, J. E. (1972). Temporal and spatial characteristics of selective encoding from visual displays. Perception \& Psychophysics, 12, 201-204. doi:10.3758/ BF03212870

Goldsmith, M., \& Yeari, M. (2003). Modulation of object-based attention by spatial focus under endogenous and exogenous orienting. Journal of Experimental Psychology. Human Perception and Performance, 29, 897-918. doi:10.1037/ 0096-1523.29.5.897

Ho, M. (2011). Object-based attention: Sensory enhancement or scanning prioritization. Acta Psychologica, 138, 45-51.

Hollingworth, A., Maxcey-Richard, A. M., \& Vecera, S. P. (2012). The spatial distribution of attention within and across objects. Journal of Experimental Psychology. Human Perception and Performance, 38, 135-151. doi:10.1037/ a0024463

Kasai, T. (2010). Attention-spreading based on hierarchical spatial representations for connected objects. Journal of Cognitive Neuroscience, 22, 12-22. doi:10.1162/jocn.2008.21158

Kliegl, R., Wei, P., Dambacher, M., Yan, M., \& Zhou, X. (2010). Experimental effects and individual differences in linear mixed models: Estimating the relationship between spatial, object, and attraction effects in visual attention. Frontiers in Psychology, 1, 238. doi:10.3389/fpsyg.2010.00238

Law, M. B., \& Abrams, R. A. (2002). Object-based selection within and beyond the focus of spatial attention. Perception \& Psychophysics, 64, 1017-1027. doi:10.3758/BF03194753

Martinez, A., Ramanathan, D. S., Foxe, J. J., Javitt, D. C., \& Hillyard, S. A. (2007). The role of spatial attention in the selection of real and illusory objects. Journal of Neuroscience, 27, 7963-7973. doi:10.1523/JNEUROSCI.0031-07.2007

Moore, C. M., \& Fulton, C. (2005). The spreading of attention to hidden portions of occluded surfaces. Psychonomic Bulletin \& Review, 12, 301-306. doi:10.3758/BF03196376

Moore, C. M., Yantis, S., \& Vaughan, B. (1998). Object-based visual selection: Evidence from perceptual completion. Psychological Science, 9, 104-110. doi:10.1111/14679280.00019

Mozer, M. C. (2002). Frames of reference in unilateral neglect and visual perception: A computational perspective. Psychological Review, 109, 156-185. 
Müller, N. G., \& Kleinschmidt, A. (2003). Dynamic interaction of object- and space-based attention in retinotopic visual areas. Journal of Neuroscience, 23, 9812-9816.

Posner, M. I., Snyder, C. R., \& Davidson, B. J. (1980). Attention and the detection of signals. Journal of Experimental Psychology. General, 109, 160-174. doi:10.1037/0096-3445.109.2.160

Richard, A. M., Lee, H., \& Vecera, S. P. (2008). Attentional spreading in object-based attention. Journal of Experimental Psychology. Human Perception and Performance, 34, 842-853. doi:10.1037/00961523.34.4.842
Shomstein, S., \& Behrmann, M. (2008). Object-based attention: Strength of object representation and attentional guidance. Perception \& Psychophysics, 70, 132-144. doi:10.3758/ PP.70.1.132

Shomstein, S., \& Yantis, S. (2002). Object-based attention: Sensory modulation or priority setting? Perception \& Psychophysics, 64, 41-51. doi:10.3758/BF03194556

Shomstein, S., \& Yantis, S. (2004). Configural and contextual prioritization in object-based attention. Psychonomic Bulletin \& Review, 11, 247-253. doi:10.3758/BF03196566 